\title{
Reduction Effect of Traffic Accidents by Function of Drowsiness Detection
}

\author{
Masahiro Miyaji \\ The Institute of Information Science and Technology, Aichi Prefectural University, Japan
}

Copyright $(2016$ by authors, all rights reserved. Authors agree that this article remains permanently open access under the terms of the Creative Commons Attribution License 4.0 International License

\begin{abstract}
Drowsiness is thought as crucial risk factor which may result in severer traffic accidents. Recently driver's psychosomatic state adaptive driving support safety function has been highlighted to further reduce the number of traffic accidents. Consequently, reduction effect of psychosomatic adaptive safety function should be clarified to foster its penetration into commercial market. This research clarified root cause of traffic incidents experiences by means of introducing Internet survey. From statistical analysis of the traffic incidents experiences, major psychosomatic state just before traffic incidents was identified as haste, distraction and drowsiness. This research focused drowsiness of a driver while driving. By means of using the Kohonen neural network, this research created estimating accuracy to detect a state of drowsiness. As a self-organized map, this research introduced six types of facial expression. Finally, this research estimated reduction effect of driver's drowsiness in the traffic accident. Result of the estimation was verified by comparing to the reduction effect of ESC.
\end{abstract}

Keywords Traffic Accident Reduction, Drowsiness, Kohonen Neural Network, ASV, ITS

\section{Introduction}

The number of traffic fatalities in Japan as of 2014 has declined under 4,200 and the number of traffic accidents has declined as shown in Figure 1 [1]. However, the number of traffic injuries has still exceeded 0.7 million, reducing the number of traffic accident has become an inevitable challenge to establish sustainable mobile society. Consequently, creating technologies which may reduce traffic accidents remains one of the highest ranked issues. From the reason preventive safety technologies may play more and more important role, one of candidates may be driver's psychosomatic state adaptive driving support function, which has potential ability to enhance safety performance of vehicle safety [2] [3]. Accordingly, identifying root cause of traffic accidents may be indispensable. The Safety Engineering Committee of the
Science Council of Japan (2000) [4] has asserted that analytical survey of traffic accidents as well as traffic incidents is essential to clarify root cause of traffic accidents. Previous research reported that $71 \%$ to $90 \%$ of traffic accidents were occurred by human factors [5] [6] [7].

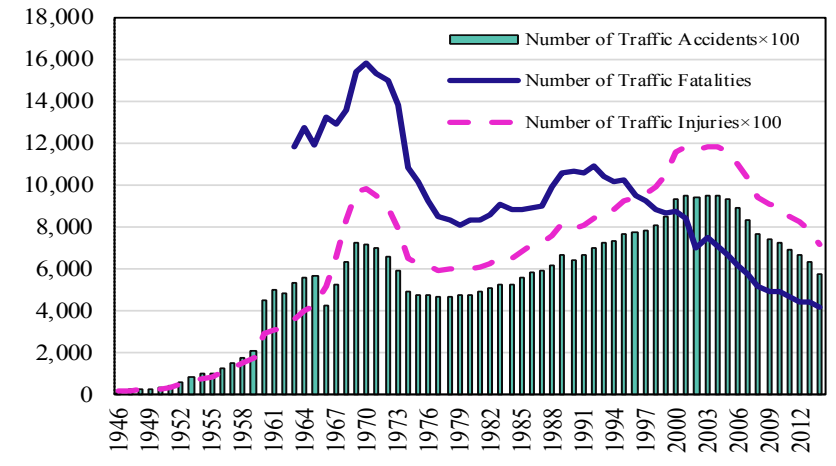

Figure 1. Trends of road traffic accidents as of 2014 in Japan

This research assumed that root cause of traffic accidents may be the same as that of traffic incidents including near-miss accidents. According to the previous research of the author [8], this research revised the outcome of Internet survey which used questionnaire because of its easiness to collect real world experiences. In the previous research seven traffic incidents model were defined by referring seven traffic accidents model used in the Advanced Safety Promotion Project Phase III in Japan (ASV) [9]. Survey area of traffic incidents experiences was extended to ordinary driving occasion which has potential accident risk as well as apparent accident risk as shown in Figure 2.

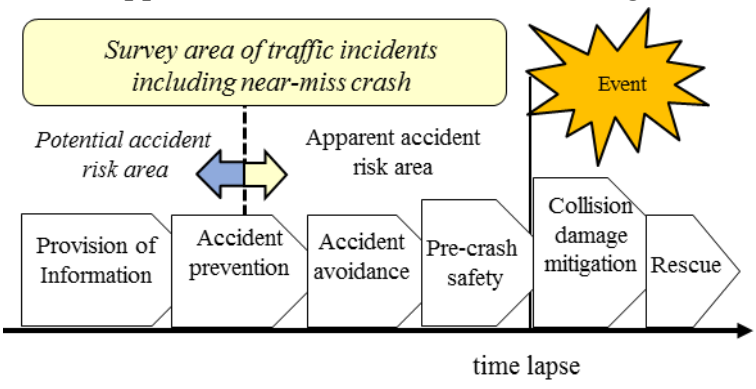

Figure 2. Survey area of traffic incidents 
From the analysis of collected traffic incidents experiences, major psychosomatic states were identified as hasty, distraction and drowsiness. This research focused driver's drowsiness which may result in severer traffic accidents. Lots of the research was done to detect drowsiness. One approach is to relate physiological states with drowsiness, which are percent eye closure (PERCLOS) [10], [11], Electrocardiogram (ECG) [12], and blinking [13]. In the previous research of author, Herat rate variability (HRV) was used to predict sleepiness onset [14]. However, those methods are not enough to estimate detection accuracy of drowsiness because accuracy of drowsiness detection is essential to estimate reduction rate of traffic accidents. Another approach is to use facial expression for estimation of drowsiness. One previous research used facial expression in combination with subjective sleepiness [15]. But this research could not directly estimate accuracy of drowsiness detection. From the previous research [16], facial expression is expressed by using Kohonen neural network (hereinafter; KNN) [17]. In the previous research of the author [18], recognition of six types of facial expressions was identified by using KNN which created a self-organized map of drowsiness. Normalization of orientation and size of face was executed by coordination of eyes and nose. Facial expression was judged by minimization of Mahalanobis' distance as a recognition algorithm. Then this research established to detect drowsiness of a driver in higher accuracy. Finally, reduction rate of the number of traffic accidents was estimated by means of using amount of average accuracy of detecting drowsiness. This research compared reduction rate of drowsiness detection function with the reduction rate of ESC by using database of National Agency for Automotive Safety \& Victims' Aid in Japan [19] and US-DOT [20].

\section{Identification of Root Cause of Traffic Incidents Experiences}

In revised Internet survey of the author, the number of respondents was 2,000 (1,117 males and 883 females), the average age was 41.1 years, the average driving experience was 19.9 years and the average number of traffic incidents was 2.34. Statistical sampling error of the Internet surrey was calculated by $2.2 \%$. Revised analytical results of the survey were shown in Figure 3.

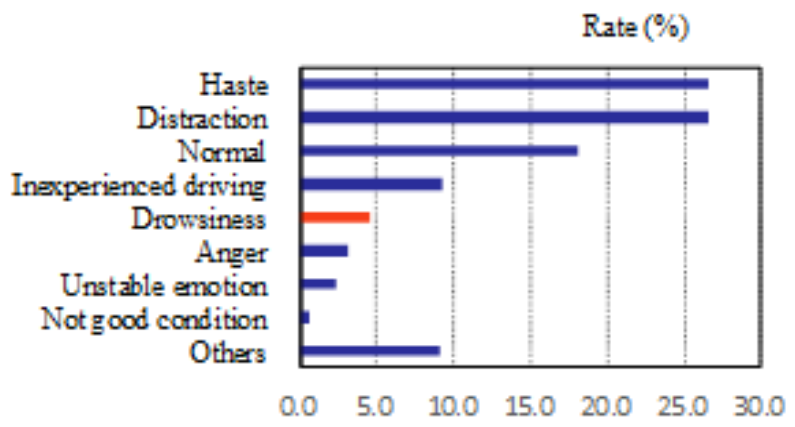

Figure 3. Psychosomatic states just before traffic incidents (Units: \%)
Top four psychosomatic states just before traffic incidents were "Hasty" (26.6\%), "Distraction" (26.5\%), and "Normal" $(18.0 \%)$ as well as "Drowsiness" $(4.6 \%)$. This result of drowsiness agreed with previous research [21]. From the results detecting driver's psychosomatic state just before traffic incident is indispensable for establishing countermeasures to reduce the number of the traffic accident. This study focused a state of drowsiness as driver's psychosomatic state to reduce severer traffic accidents.

\section{Creation of Facial Expression Map}

Kohonen neural network is known as one of competitive learning type neural network which only winning neuron can learn input data set in learning stage. This learning may have feature to create self-organized map which expresses correlation among input data of facial expression. Consequently, Kohonen neural network may generate complementary picture among each facial expressions. Previous research reported that facial expression of humankind which shows emotion were six types [22]. They were "ordinary", "drowsiness", "anger", "sorrow", "delight", "surprise". Accordingly, this research used the above six facial expressions. Six pictures were taken for each six facial expressions. Original face expression in $512 \times 512$ pixel of 24 bit was gray-scaled. Then both orientation and dimension of face were corrected by coordination eyes. A picture in $256 \times 256$ pixel of facial expression was extracted by referring central point of nose. Then this picture was compressed in $64 \times 64$ pixel. Accordingly, self-organized map was created as input vector of Kohonen neural network. Output picture of Kohonen neural network unit was in $32 \times 32$ pixel.

\section{Recognition of Facial Expression}

By using Mahalanobis' distance as a recognition algorithm, this research tried to estimate accuracy of detecting drowsiness of voluntarily participants. The number of participants was 8 , who consented to join in this experiment. Distribution degree of similarity was acquired from generated map. Accordingly, strongly reacted portion to a maximum similarity data was extracted by a certain amount of Mahalanobis' distance. Then recognition was executed whether the portion was included following equation (1).

$$
P_{i}=\sum_{k}^{N_{i}}\left(x_{i k}-x_{\min }\right)
$$

In equation (1), $1_{i}$ denotes class of facial expression, $P_{i}$ denotes similarity of class of facial expression in the same distribution, $x_{j k}$ denotes similarity of data $k$ in $i$ class of facial expression, $x_{\min }$ denotes minimum similarity on similarity map. Consequently, accuracy of facial expression was defined by maximum value of facial expression class derived from equation (1). 


\section{Recognition Results}

This research obtained cooperation of 8 participants who consented to join the experiment. This research took 6 pictures for 6 facial expressions per one participant. 240 out of 288 picture of facial expression was selected. 40 facial expressions were allocated for each facial expression. Then recognition experiment by means of using Kohonen neural network was executed. At the same time subjective evaluation by six facial expressions was executed by the same participant. Accordingly, average recognition accuracy of facial expression was obtained as shown in Table 1 . Recognition accuracy of drowsiness showed maximum value among 6 facial expressions, which was $93.8 \%$ in top common. However, amount of subject evaluation of drowsiness was in fourth top common. Average value of recognition accuracy of these 6 facial expressions was almost the same amount as subjective evaluation. Consequently, this method by means of using Kohonen neural network was concluded as suitable in use to estimate a state of drowsiness. Then this research selected $87.6 \%$ as average drowsiness detection accuracy between facial expression and subjective evaluation.

Table 1. Recognition accuracy of facial expression (Units; \%)

\begin{tabular}{|c|c|c|}
\hline $\begin{array}{c}\text { Type of facial } \\
\text { expression }\end{array}$ & $\begin{array}{c}\text { Recognition } \\
\text { accuracy (\%) }\end{array}$ & $\begin{array}{c}\text { Subjective } \\
\text { evaluation (\%) }\end{array}$ \\
\hline Ordinary & 75.0 & 97.9 \\
\hline Drowsiness & $\mathbf{9 3 . 8}$ & $\mathbf{8 1 . 3}$ \\
\hline Anger & 81.3 & 91.7 \\
\hline Sorrow & 60.4 & 39.6 \\
\hline Delight & 91.7 & 89.6 \\
\hline Surprise & 87.5 & 93.8 \\
\hline Average & 84.2 & 82.3 \\
\hline
\end{tabular}

\section{Estimation of Reduction Rate of Function of Drowsiness Detection}

Table 2. Reduction rate of the number of traffic accidents by ASV system (Units; \%)

\begin{tabular}{|c|c|c|c|}
\hline Vehicle type & $\begin{array}{c}\text { Ratio of } \\
\text { owned vehicle }\end{array}$ & $\begin{array}{c}\text { Reduction rate } \\
\text { of traffic } \\
\text { accident }\end{array}$ & \multirow{2}{*}{$\begin{array}{c}\text { Overall } \\
\text { reduction rate }\end{array}$} \\
\cline { 1 - 2 } Passenger & 75.1 & 41.8 & \multirow{2}{*}{38.7} \\
\cline { 1 - 2 } Heavy duty & 20.4 & 28.4 & \\
\hline Motor cycle & 4.5 & 47.3 & \\
\hline
\end{tabular}

* Whole number of owned vehicle: 80,320,157 as of Jan. 2015

This research estimated reduction rate of the number of traffic accident by using above amount of average accuracy of recognizing drowsiness state. Reduction rate of the number of traffic accident by using preventive safety system (Advanced Safety Vehicle; ASV) was adopted as shown in Table 2 from the research which number was released on vehicle type basis by the MLIT in Japan [23].

Overall reduction rate of preventive safety systems was calculated to $38.7 \%$ in weighted average as shown in Table 2 as of Jan. 2015. The number derived in this research showed almost the same amount as the number released by the US-DOT, which is $35 \%$ in overall for Intelligent Transportation Systems. The number derived in this research may be judged suitable in use for estimation of reduction rate of the number of the traffic accident for preventive safety systems. Accordingly reduction rate of the number of the traffic accident by incorporating the driver states monitoring function as preventive safety unit may be estimated in following steps;

A. Define $R_{d}$ as reduction rate of the number of traffic accident based on function of drowsiness detection, where this research used average accuracy of drowsiness by facial expression detection.

B. $R t$ is defined as reduction rate of the number of traffic accident by ASV system as shown in Table 2 (38.7\%).

C. $D_{r}$ is defined as constituent ratio of the driver's drowsiness state $(4.6 \%)$.

D. $D_{d}$ is defined as average detection accuracy of drowsiness obtained by Kohonen neural network based on facial expressions (87.6\%).

Consequently, $\mathrm{R}_{\mathrm{d}}$ may be calculated following equation (2).

$$
\mathrm{R}_{\mathrm{d}}=R_{t} \times D_{r} \times D_{d}
$$

When the amount is set as $R_{t}=0.387, D_{r}=0.046$ and $D_{d}=$ $0.876, \mathrm{R}_{\mathrm{d}}$ can be calculated to 0.016 for overall traffic accidents. Although some statistics for reduction rate of preventive safety devices have been analyzed, this research selected ESC analytical case. The reduction rate of the number of the traffic accident by ESC was identified as 0.036 from the study of National Agency for Automotive Safety \& Victims' Aid (NASVA) in Japan. This research defined $I_{\text {esc }}$ as ESC installation rate in Japanese market [24]. When let $R_{\text {esc }}$ as overall reduction rate of the number of traffic accident by ESC, $\mathrm{R}_{\mathrm{esc}}$ may be expressed following equation (3).

$$
\mathrm{R}_{\mathrm{esc}}=0.36 \times I_{\text {esc }}
$$

When $I_{e s c}$ was estimated as 0.327 as of $2014, \mathrm{R}_{\mathrm{esc}}$ is calculated to 0.118 .

Reduction rate of the number of the traffic accident by using the driver's drowsiness monitoring function was estimated as $1.6 \%$, whereas that of ESC was $11.8 \%$. As amount of reduction rate of driver's drowsiness monitoring function is approximately one seventh compared with that of $\mathrm{ESC}$, its effect of reducing the number of traffic accident can be expected in a certain degree. 


\section{Conclusions}

This research identified root cause of the traffic incident by means of conducting Internet survey, which may be the same as root cause of the traffic accident. To estimate accuracy of drowsiness state, this research introduced picture recognition method which uses six facial expressions, which used Kohonen neural network as recognition algorithm. Finally, this research created novel method of estimating reduction rate of the traffic accident by means of using driver's drowsiness detection function. The conclusions are as follows;

A. Drowsiness is one of major driver's psychosomatic states just before traffic accidents which is likely to be involved in severer traffic accidents.

B. Internet survey of traffic incidents in the real field may be one of effective means to identify root cause of traffic accidents on big data basis.

C. Kohonen neural network may be effective means to estimate an accuracy of drowsiness state as well as anger. The method by using facial expression is unique means to detect accuracy of drowsy state.

D. Reduction effect by using driver's drowsiness detection function is expected in certain degree compared with that of ESC. Consequently, wider installation of drowsiness detection function should be incorporated into production vehicle to avoid traffic accidents.

Future issue includes further enhancing performance of detecting driver's drowsiness as well as anger. Moreover, statistical investigation including Internet survey for reduction rate of the number of the traffic accidents in the real world would be widely executed to promote introduction of vehicle safety devices for the reduction of the number of the traffic accidents.

\section{Acknowledgements}

Hearty appreciation for the dedicated support from $\mathrm{T}$. UMEZAKI, Professor of the Graduate School of Engineering, Nagoya Institute of Technology and also Project Professor of Interfaculty Initiative in Information Studies of the University of Tokyo in Japan, and, K. OGURI, Professor of the Graduate School of Information Science and Technology, Aichi Prefectural University in Japan, and H. KAWANAKA, Associate Professor of the Graduate School of Information Science and Technology, Aichi Prefectural University in Japan.

\section{REFERENCES}

[1] National Police Agency in Japan (2015). Road traffic accidents as of 2014. Retrieved from http://www.e-stat.go.jp/ SG1/estat/List.do?lid=000001128896/
[2] A. Hattori, S. Tokoro, M. Miyashita. Development of forward collision warning system using the driver behavioral information, SAE TECHNICAL PAPER SERIES, 2006 SAE World Congress, Vol. No.115, Section 7, 818-827, 2006.

[3] T. Nishina, K. Moriizumi. Development of new pre-crash safety system using driver monitoring sensor, 15th World Congress on ITS, TS135-10315, 1-12, 2008.

[4] The expert committee for safety engineering in Science Council of Japan. A Suggestion by the Science Council of Japan about Accident Investigation Method, The Proposal Report of the Science Council of Japan, March 27, 2000, Science Council of Japan, 7-22-34, Roppongi, Minato-ku, Tokyo, JAPAN 106-85559

[5] J. R. Treat, N. S. Tumbas, S. T. McDonald, D. Shinar, R. D. Hume, R.E. Mayer, and R. L. Stasifer. Tri-level Study of the Causes of Traffic Accidents, US-DOT-HS-034-3-535-77, Indiana University, 1977.

[6] MLIT in Japan on Road Bureau. Background of AHS R\&D, http://www.mlit.go.jp/road/ITS/, 2014.

[7] S. G. Klauer, T. A. Dingus, V. L. Neale, J. D. Sudweeks, and D. J. Ramsy. The Impact of Driver Inattention on Near-Crash/Crash Risk: An Analysis Using the 100-Car Naturalistic Study Data, US-DOT-HS-810-594, April, 2004.

[8] M. MIYAJI, M. DANNO, K. OGURI. Analysis of Driver Behavior based on Experiences of Road Traffic Incidents Investigated by means of Questionnaires for the Reduction of Traffic Accidents, International Journal of ITS Research, Vol.6, No.1, 47-56, 2008.

[9] R. Toji. Advanced Safety Vehicle Promotion Project: Phase3 and 4. Journal of Automotive Engineers of Japan, Vol. 60, No. $12,10-13,2006$

[10] Paul Rau. Drowsy Driver Detection and Warning System for Commercial Vehicle Drivers: Field Operational Test Design, Analysis, and Progress, SAE Government / Industry Meeting, May 11, 2005

[11] L. M. Bergasa, J. Nuevo, M.A. Sotelo, R. Barea and M.E. Lopez. Real-Time System for Monitoring Driver Vigilance, IEEE TRANSACTIONS ON INTELLIGENT TRANSPORTATION SYSTEMS, Vol. 7, No.1, March 2006.

[12] C. Papadeils, Zhe Chen, C. K. Papadeli, P. D. Bamidis, I. Chouvarda, E. Bekiaris and N. Maglaveras. Monitoring sleepiness with on -board electrophysiological recordings for preventing sleep-deprived traffic accidents, Clinical Neurophysiology, Vol. 118, Issue 9, 1906-1922, September 2007

[13] A. Pict, S. Charbonnier and A. Caplier. Drowsiness detection based on visual signs: blinking analysis based on high frame rate video, IEEE International Instrumentation and Measurement Technology Conference, Austin Texas, USA, 801-804, May 2010.

[14] A. Noda, M. Miyaji, Y. Wakuda, F. Yasuma, T. Fukuda, K. Iwamoto and N. Ozaki. Simultaneous Measurement of Heart Rate Variability and Blinking Duration to Predict Sleep Onset and Drowsiness in Drivers, Journal of Sleep Disorders \& therapy, Vol. 4, Issue 5, 1-3, September 2015.

[15] S. Hachisuka. Human and Vehicle-Driver Drowsiness Detection by Facial Expression, International Conference on Biometrics and Kansei Engineering (ICBAKE) 2013, 
320-326, Tokyo, Japan, July 2013.

[16] S. Hirano, T. Umezaki and Y. Sato. Automatic generation of facial expressions in three dimension in Japanese, IEICE Technical Report, The Institute of Electronics, Information and Communication Engineer, PRMU99-135, 13-20, November, 1999.

[17] T. Kohonen. Self-Organizing Map, Spriber, Berlin, Heiderberg, 1955.

[18] H. Kato, T. Umezaki, M. Danno and M. Miyaji. Recognition of facial expressions by using Kohonen based Neural Network in Japanese, Proceedings of 13th Symposium on Sensing via Image Information, IN2-05, 1-4, June 2007, Yokohama, Japan.

[19] National Agency for Automotive Safety \& Victims' Aid
(NASVA) in Japan. Effect of ESC, February 18, 2005.

[20] US DOT. ITS Benefit Database, http://www.itsbenefits.its.dot .gov/its/benecost.nsf/

[21] D.F. DIGES. An overview of sleepiness and accidents, Journal of Sleep Research, Vol. 4, 4-14, 1995

[22] P. Ekman. Facial expression and emotion, American Psychologist, Vol. No. 48(4), 384-392, April 1993.

[23] MLIT in Japan on Road Bureau. Estimation of reduction effects of traffic accidents by ASV technology as of 2014, http://www.mlit.go.jp/jidosha/anzen/01asv/japanese/asv2effe ct.html

[24] H. Baum, T. Fujiwara, Y. Kidokoro. Analysis of Cost/ Benefit for ESC, Policy Information Centre of the National Graduate Institute in Japan for Policy Studies (GRIPS), 2009 